\title{
3D Photonic Crystals from highly monodisperse FRET- based red luminescent PMMA spheres
}

\author{
J. F. Galisteo-López, ${ }^{*} \uparrow$ M. Ibisate, A. Muñoz and C. López \\ Instituto de Ciencia de Materiales de Madrid (CSIC), c/ Sor Juana Inés de la Cruz 3,
}

28049 Madrid (Spain)

\begin{abstract}
Red-luminescent PMMA spheres containing a Förster resonance energy transfer (FRET) pair were synthesized via a two-step polymerization method. Two reaction parameters, time and monomer volume, are scanned in order to tune the sphere diameter in the $250-500 \mathrm{~nm}$ range. Further the polydispersity of the spheres is kept low, at ca. 3\%, regardless of sphere diameter or dye concentration. A thorough optical characterization via spectroscopy and time resolved measurements shows a FRET efficiency of over $40 \%$ before concentration quenching effects take place, allowing for a precise tuning of their emission in the red region of the visible spectrum. The high quality of these spheres makes them suitable to fabricate selfassembled 3D photonic crystals which act as photonic environment to modify the spectral properties of the FRET pair via Bragg diffraction.
\end{abstract}

\footnotetext{
$\uparrow$ Present address: Instituto de Ciencia de Materiales de Sevilla (CSIC), c/Américo Vespucio 49, 41092 Sevilla (Spain)

*e-mail address: juan.galisteo@csic.es
}

\section{Introduction}

Encapsulation of light sources within a polymeric or inorganic nano-particle (NP) provides a means to shield them from the environment improving their photophysical properties and, for certain emitters such as quantum dots, avoiding toxicity issues in their use for bio-imaging purposes. Recently the incorporation of two or more types of emitters within the nanoparticle has been eagerly explored as a way to improve its emission properties. In this approach energy from an excited emitter can be nonradiatively transferred via Förster resonance energy transfer (FRET) to a nearby (2-10 $\mathrm{nm}$ ) ground-state acceptor given a spectral overlap between their absorption and emission spectra exists and their transition dipole moments are not orthogonal. [1] The efficiency of the process is then dictated by the emitter distribution within the NP which in many cases can be controlled through their relative concentration. In this way, varying 
the ratio between the two chromophores allows producing spheres with a common excitation wavelength but different photoluminescence spectra, paving the way for multiplexed biological detection. Some examples include dye-doped nanospheres of different nature including both inorganic, [2,3] organic [4,5,6,7] and hybrid matrices. $[8,9]$

Beyond the field of bio-imaging, applications benefiting from FRET are growing and span from enhanced energy harvesting in photovoltaics [10,11] to sensing [12] or photonics (where FRET can be used as a mechanism to improve the performance of devices such as OLEDs [13]). Regarding the use of FRET NP, dye doped polymer beads have been recently used as gain media for efficient photostable red-emitting dye-lasers. [14] Further if a properly designed photonic environment is provided, the efficiency of the FRET process may be controlled via the local density of photonic states (LDOS) at the dye's location. [15] If the spheres satisfy a number of requirements such as low polydispersity, diameter in the submicron range and colloidal stability, they can be arranged in a periodic fashion to constitute themselves the photonic environment in the shape of self-assembled photonic crystals $(\mathrm{PhC})$ or artificial opals. [16] These structures have been long used as platforms to control the luminescent properties of emitters. [17] Such control can be achieved either by relying on the above mentioned LDOS control at the emitter position or by modifying the directionality of the emission through Bragg diffraction, introducing a filter effect. Recently, a few examples exploring self-assembled $\mathrm{PhC}$ to modify FRET have been presented using the artificial opal as a passive scaffold. $[18,19,20]$ This has been done impregnating the opaline matrix with well controlled donor-acceptor configurations [18] or with a random suspension of two dyes, [19] and also employing an inverse opal configuration with a rare-earth based matrix. [20] Further, the combined use of artificial opals and FRET has been demonstrated as a means to develop DNA sensors [21] or systems for optical storage. [22]

But to date no evidence of opal based PhC with FRET pairs within the spheres has been shown. Among the advantages of this configuration lies the possibility of achieving a large load of emitters since the spheres represent $74 \%$ of the total $\mathrm{PhC}$ volume and a protected environment where intimate contact or proximity is granted to the active species. If one wishes to modify the energy transfer within the NPs by organizing them into a periodic array one must fabricate highly monodisperse NP with a diameter of the order of the emission of the donor species which, for the case of the visible, translates into a few hundred nanometers. To date most of the approaches for NP fabrication have focused on diameters in the range of tens of nm (appropriate for their use in bio-imaging and other applications where emission is not meant to be modulated by the environment) presenting a large polydispersity, owing to the difficulty in synthesizing them with due size control, and monodisperse spheres have only appeared with diameters close to $2 \mu \mathrm{m}$, too large for a proper control of the emission in the visible range. If one wishes to exert a control on the energy transfer within the nanospheres using the $\mathrm{PhC}$ environment, photonic features must be present which demand a low polydispersity of the spheres. [23] The critical role of monodispersity is evident by the fact that for values above $5 \%$ crystallization of artificial opals is not expected to take place. [24]

In this work we demonstrate a method to fabricate highly monodisperse polymeric spheres doped with two dyes of the rhodamine family for their use as FRET-based luminescent media. Spheres with different dye loads are prepared with a fine control on their diameters in the 200$500 \mathrm{~nm}$ range and having a polydispersity of $c a .3 \%$. Their optical response is studied by means of photoluminescence as well as time resolved measurements demonstrating a FRET efficiency of up to $50 \%$. Their high quality renders them appropriate for their use as building blocks to 
fabricate three dimensional (3D) $\mathrm{PhC}$ in the shape of artificial opals with different lattice parameter. We show how the luminescent properties of the spheres can be further modified in these structures by means of Bragg diffraction. These results pave the way for future unconventional light sources such as lasers combining the cavity-less lasing mechanisms characteristic of 3D PhC [25] with the advantages of FRET-based lasers. [26]

\section{Experimental}

\section{Sphere synthesis:}

To remove the inhibitor, Methylmethacrylate (MMA) (Sigma-Aldrich) was washed with aqueous sodium hydroxide solution $(1 \mathrm{M})$, dried over anhydrous magnesium sulfate and finally passaged downward through a glass column containing silica gel. Water was purified through a MilliQ purification system. The rest of reagents were used without further purification. Sodium hydroxide, magnesium sulfate, silica gel 60 , potassium persulfate (KPS) and rhodamine B $(\mathrm{RhB})$ were provided by Sigma-Aldrich. LD-700 perchlorate (Rh700) was acquired from Exciton and ethanol from Panreac.

Dye doped spheres with a 250-380 $\mathrm{nm}$ diameter were fabricated using a modified version of the procedure given in reference [5]. In a $250 \mathrm{~mL} 3$-neck round-bottom flask equipped with a condenser and a gas inlet, $140 \mathrm{~mL}$ of water and the corresponding MMA volume (from 10 to 25 $\mathrm{mL}$ ) were added. The mixture was deoxygenated by bubbling nitrogen gas at room temperature for $45 \mathrm{~min}$ and then tempered for $60 \mathrm{~min}$ at $80{ }^{\circ} \mathrm{C}$. To start the polymerization $5 \mathrm{~mL}$ of a deoxygenated KPS water solution $(0.1 \mathrm{~g} / \mathrm{mL})$ were added under magnetic stirring. 5 min after the polymerization starts, $9 \mathrm{~mL}$ of a dye solution were added at a constant rate of $0.67 \mathrm{~mL} / \mathrm{min}$. The dye solution contained $6.2 \mathrm{mg}$ of RhB with different amounts of Rh700 (to obtain molar ratios from 1:1 to $1: 4 \mathrm{RhB}: \mathrm{Rh} 700)$ in a $1: 1(\mathrm{v} / \mathrm{v})$ ethanol:water mixture. The reaction time was fixed to $40 \mathrm{~min}$, except for the sample made from $25 \mathrm{~mL}$ of MMA whose reaction time was $2 \mathrm{~h}$.

In order to increase the NPs size beyond $380 \mathrm{~nm}$ a two-stage dispersion polymerization was conducted. In this procedure the previous protocol was followed fixing the initial volume of MMA at $25 \mathrm{~mL}$ and adding an extra MMA volume $(10,15$ or $20 \mathrm{~mL}$ at a rate of $0.67 \mathrm{~mL} / \mathrm{min})$ immediately after the dyes solution addition. The reaction was allowed to proceed for $2 \mathrm{~h}$, longer times leading to aggregation of the spheres. As dyes were added once the reaction was initiated, the grown PMMA spheres will have a few nm sized core without any dye in it. In addition an increasing gradient in the dye concentration will take place radially across the PMMA NP due to the slow dye addition to the reaction flask. Transmission Electron Microscopy (TEM) images were taken in order to estimate the diameter of the grown spheres using a JEOL 2000 FX II model. Statistics were derived from 100 spheres for each of sample.

\section{Optical characterization:}

Optical characterization was carried out by means of photoluminescence (PL) spectroscopy and time resolved measurements. The samples were all measured under the same conditions. Quartz cuvettes with a $1 \mathrm{~mm}$ optical path containing a NP aqueous solution with fixed sphere concentration $(1 \mathrm{mg} / \mathrm{mL})$ were used for all acceptor to donor ratios $Q_{\mathrm{AD}}$. The concentration was kept low in order to avoid scattering from the spheres influencing the measurements. Optical pumping was performed with a tunable pulsed laser (OPerA-Solo from Coherent) having a repetition rate of $1 \mathrm{kHz}$ and delivering $150 \mathrm{fs}$ long pulses. The pump wavelength $(515 \mathrm{~nm})$ was chosen in order to maximize optical excitation of the donor while minimizing that of the 
acceptor. PL spectra were collected with a fiber coupled spectrometer USB2000 (Ocean Optics). Time resolved measurements were performed (with a resolution of $80 \mathrm{ps}$ ) with a Time Correlated Single Photon Counting card (SPC-300 from Becker \& Hickl) at a fixed wavelength selected with a monochromator.

\section{Artificial opal fabrication and characterization:}

Thin film artificial opals were fabricated by vertically placing a clean glass substrate into a 20 $\mathrm{mL}$ vial containing a $0.1 \%$ vol. aqueous dispersion of spheres. The dispersion was left in a humidity $(60 \%)$ and temperature $\left(45^{\circ} \mathrm{C}\right)$ controlled chamber for 24 hours. Normal incidence reflectance spectra were collected using a FTIR spectrometer (Bruker IFS-66/S) coupled to an optical microscope with a $10 \times$ objective $(\mathrm{NA}=0.12)$. PL spectra from the opals were collected in an inverted microscope using a high NA objective $(\mathrm{NA}=0.75)$ as focusing/collection optics.

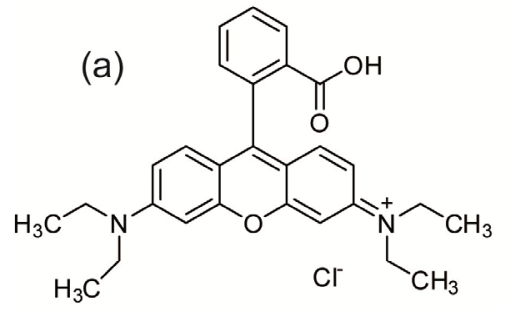

(b)
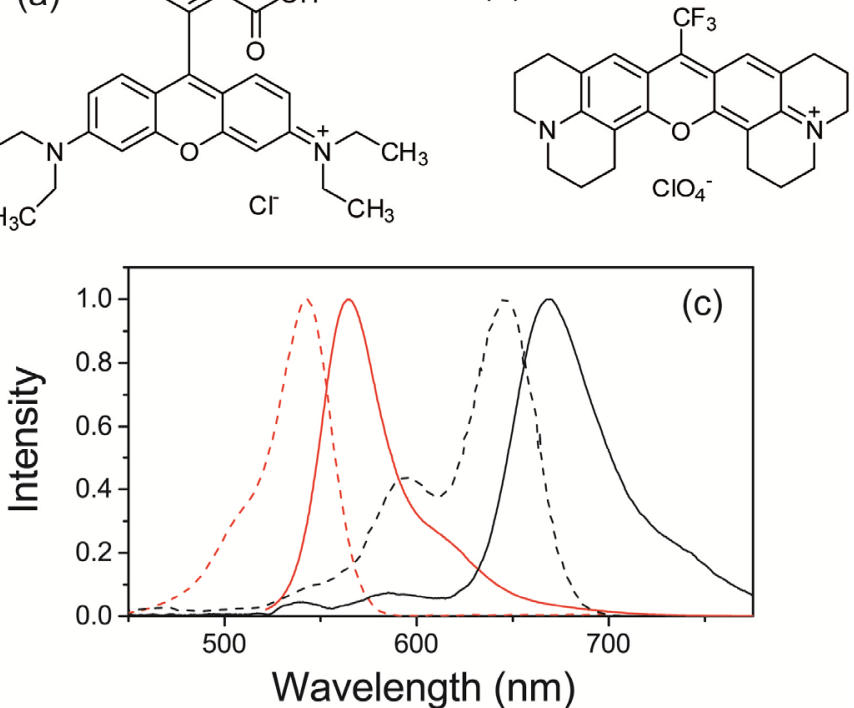

Fig. 1 Chemical structure of donor (a) and acceptor (b) molecules. (c) Absorption (dashed) and PL (solid line) spectra of donor (red) and acceptor (black line).

\section{Results}

The dyes selected as donor and acceptor were $\mathrm{RhB}$ and $\mathrm{Rh} 700$ (see Figure 1) which satisfy a number of conditions: they could be individually introduced into PMMA spheres (up to a maximum concentration of $0.8 \mathrm{mg} / \mathrm{mL}$ of MMA) before concentration quenching took place, the donor can be optically pumped using wavelengths for which the polymeric matrix is transparent and there is a good spectral overlap between donor emission and acceptor absorption. Further, the spectral overlap between the absorption bands of the species is small enough as to grant a selective excitation of the donor alone (vide infra).

As mentioned above two parameters were changed in order to control the sphere diameter: monomer volume and reaction time. For a single MMA addition and 40 min reaction time, spheres in the 250-380 $\mathrm{nm}$ range were obtained changing the MMA initial volume. In order to further increase the sphere diameter up to $500 \mathrm{~nm}$ a second addition of MMA was introduced and reaction time increased to 2 hours. Figure 2 a shows the change in sphere diameter with total MMA volume for the two reaction times. Figure $2 b$ shows the size distribution for spheres fabricated using $25 \mathrm{~mL}$ of MMA in $140 \mathrm{~mL}$ of distilled water. For the sample containing only donor molecules $\left(Q_{\mathrm{AD}}=0\right)$ a sphere diameter of $383 \mathrm{~nm}$ and a polydispersity of $2.2 \%$ was obtained. Upon addition of increasing amounts of acceptor molecules the sphere quality was not affected, the polydispersity raising to just $3.7 \%$ (Figure $2 \mathrm{c}$ ). Further, changing the $Q_{\mathrm{AD}}$ ratio did 
not affect the sphere diameter and only variations of $c a .3 \%$ in sphere diameter were observed from sample to sample.
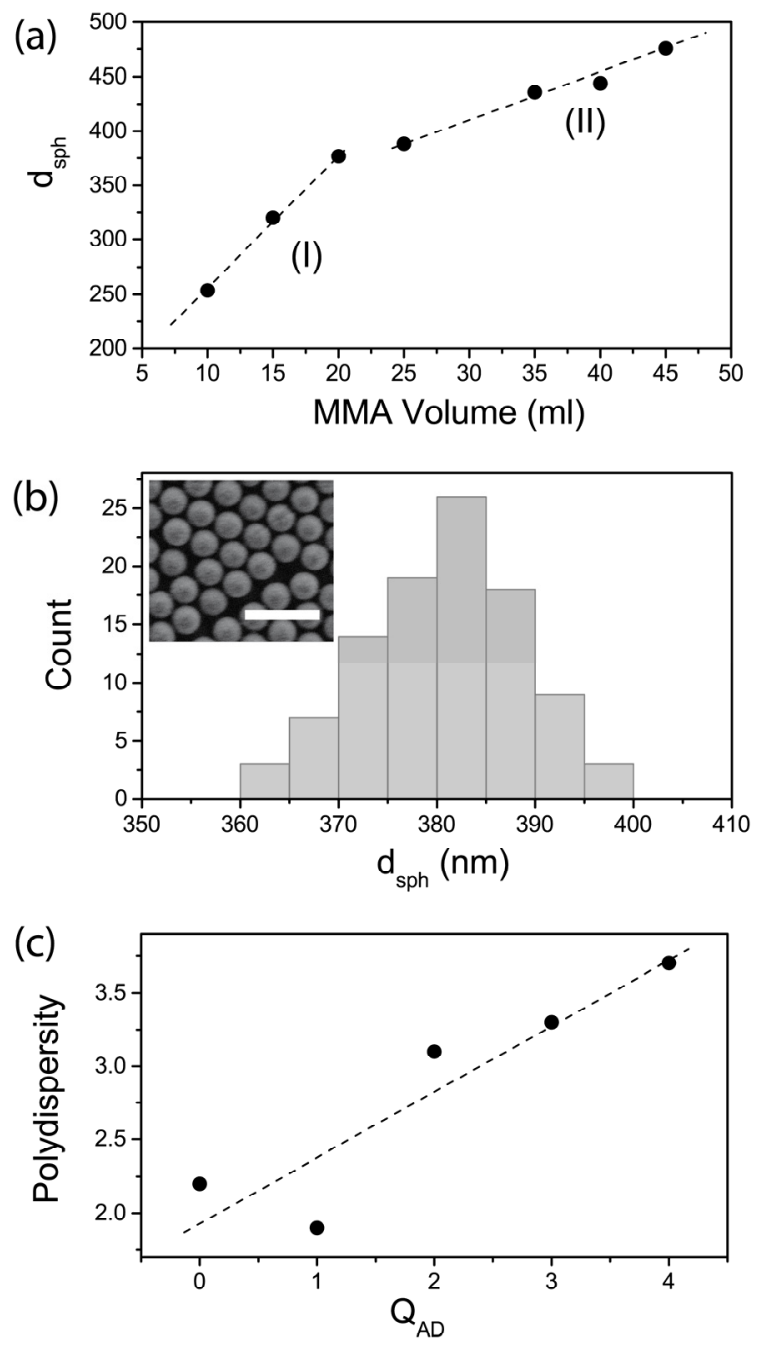

Fig. 2 (a) Dependence of sphere diameter on MMA volume. The two highlighted regions correspond to: (I) 40 minutes and (II) 2 hours reaction time. Dashed lines are guides to the eye. (b) Size distribution for spheres fabricated from $25 \mathrm{~mL}$ of $M M A$ and a ratio $Q_{A D}=0$. Inset shows an SEM image of the spheres (scale bar is 1 $\mu \mathrm{m})$. (c) Evolution of the polydispersity for spheres fabricated with $25 \mathrm{~mL}$ of MMA and increasing $Q_{A D}$ ratio.

Next we optically characterized the spheres employing PL spectroscopy as well as time resolved experiments. Figure 3 shows PL measurements for samples having a diameter of $380 \mathrm{~nm}$ (corresponding to samples grown from $25 \mathrm{~mL}$ of MMA) and a variable acceptor to donor ratio. Here we can see how for the spheres containing donor molecules only $\left(\boldsymbol{Q}_{\mathrm{AD}}=0\right)$ a PL peak centered at $577 \mathrm{~nm}$, characteristic of $\mathrm{RhB}$, is present. As we increase the acceptor concentration a PL peak centered at $670 \mathrm{~nm}$ appears associated with the emission of Rh700. 
(a)
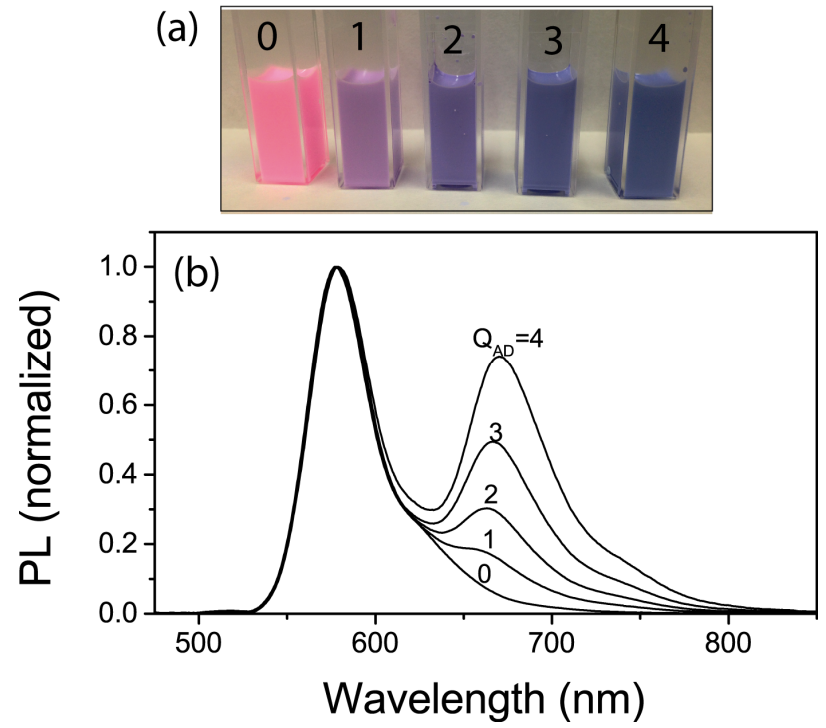

Fig. 3 (a) Aqueous suspensions and (b) normalized PL spectra of PMMA spheres fabricated from a total volume of $25 \mathrm{~mL}$ of MMA and having different $Q_{A D}$ ratios (indicated for each sample and PL curve).

In order to evaluate the influence of energy transfer and direct optical pump in the evolution of the acceptor emission, we carried out a set of control measurements. We pumped, using the same wavelength and power, a set of control samples consisting of spheres containing only acceptor molecules with identical concentration as the test samples but without donor species. Figure 4 shows the intensity of PL maxima for donor and acceptor emission (taken at 577 and $670 \mathrm{~nm}$ respectively). Here it is evident that as D emission decays, A emission increases and only about $20 \%$ of it is due to direct optical excitation, the rest being due to energy transfer from the acceptor. For values of $Q_{\mathrm{AD}} \geq 3$ we can see how the rise in A PL saturates and then decreases. This behavior points to the formation of non-emitting complexes either between donor and acceptor or between identical molecules (self-quenching) for such large dye concentrations. As a matter of fact, quenching is likely taking place between donor and acceptor molecules as for the case of the control samples (containing only acceptor molecules) the saturation behavior is less evident. A similar behavior was observed for samples with smaller spheres $(250 \mathrm{~nm})$ at a smaller ratio $\left(Q_{\mathrm{AD}}=1\right)$. For larger spheres $(480 \mathrm{~nm})$ no saturation was observed for all $Q_{\mathrm{AD}}$ ratios under consideration.

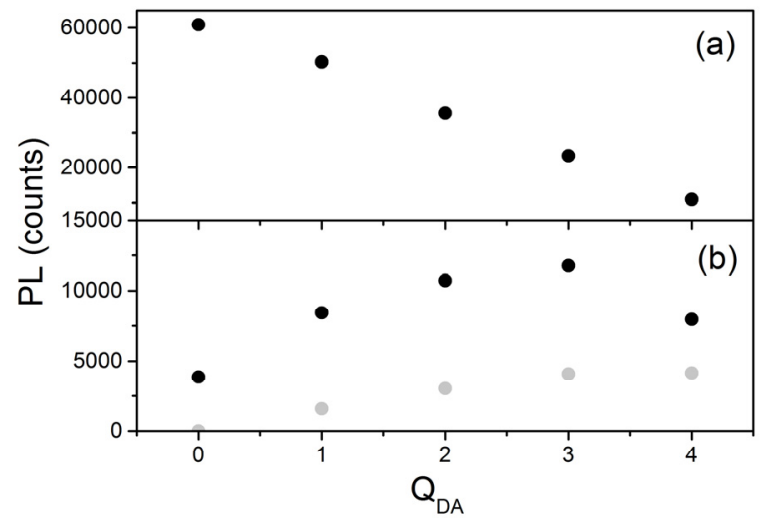

Fig. 4 Intensity at the maximum of PL for donor (a) and acceptor (b) emission corresponding to $\lambda=577$ and $670 \mathrm{~nm}$ respectively. Grey circles correspond to direct optical pumping of reference samples containing only acceptor molecules. 
To further evaluate the influence of quenching due to aggregation of the molecules within the polymer spheres, the decay dynamics of control and FRET samples were studied. In a first step a set of PMMA beads were fabricated with an increasing amount of donor dye. For RhB concentrations exceeding that used in the present work by a $c a$. four-fold factor (see Experimental section) decay dynamics of the spheres were collected at $\lambda=585 \mathrm{~nm}$. Figure 5 shows results for spheres fabricated from $25 \mathrm{~mL}$ of MMA. A behavior close to the singleexponential ideal scenario, where all molecules, once encapsulated within the PMMA sphere, are sensing the same environment was observed (see Fig. 5 a). Nevertheless, to correctly fit the data a multiexponential fit corresponding to a distribution of decay rates [27] has to be assumed. In our case, the distribution that best fitted the data was a lognormal one, as previously observed in dye-doped biopolymeric matrices. [28] The need for a multiexponential fit to describe the decay dynamics of dyes incorporated into polymeric beads is known even for commercial spheres of different size. [29] In our case, the need for a distribution of decay rates is likely related to an inhomogeneous distribution of dye molecules within the sphere intrinsic to the growth method where the dye concentration increases during the polymerization process (see above). The decay distributions for the control samples with different dye concentrations presented a small full width at half maximum (FWHM) of $\left(0.11 \pm 0.02 \mathrm{~ns}^{-1}\right)$ and a constant most frequent value $\Gamma_{\mathrm{MF}}$. The latter indicates that no additional non-radiative decay paths associated with self-quenching are taking place independent of dye concentration. The obtained decay rate for the donor within the PMMA beads $\left(0.342 \pm 0.007 \mathrm{~ns}^{-1}\right)$ was slightly larger than the value for molecules in an ethanol solution $\left(0.320 \pm 0.002 \mathrm{~ns}^{-1}\right)$ as a consequence of the different environment of the dye molecules. This behavior in the dynamics of the dyes was accompanied by a linear increase in its PL, further evidence of the absence of aggregation.

In a second set of control experiments, the dynamics of acceptor molecules was studied in FRET samples containing both dyes as the ones in Figs.3 and 4. The samples were pumped with $\lambda=633 \mathrm{~nm}$ and PL was collected at $\lambda=680 \mathrm{~nm}$ (see Fig. 5c). Under these pump conditions we are certain that acceptor molecules are not being excited via FRET. Again, experimental data could be fitted with lognormal distributions with a small FWHM of $0.03 \pm 0.01 \mathrm{~ns}^{-1}$ ) and a nearly constant most frequent decay rate of $0.31 \pm 0.01 \mathrm{~ns}^{-1}$ were obtained save from the sample having the highest acceptor load $\left(\mathrm{Q}_{\mathrm{AD}}=4\right)$ for which a faster dynamics with $\Gamma_{\mathrm{MF}}=0.36 \mathrm{~ns}^{-1}$ was obtained. Such change in the acceptor dynamics is related to aggregation in the presence of acceptor as identical samples, only containing no donor molecules (grey dot in the figure), showed a value similar to that of the FRET spheres with lower donor load.

In order to evaluate whether energy transfer between donor and acceptor is taking place via a resonant process (i.e. FRET) in the spheres containing both dyes we next studied the dynamics of the emission of the donor in the absence and presence of acceptor. If a donor molecule is sufficiently close to an acceptor one, non-radiative paths of energy transfer will be available for the former via long-range dipole-dipole interaction with the latter. The addition of non-radiative (FRET) de-excitation paths with a decay rate $\Gamma_{\text {FRET }}$ to the radiative ones $\Gamma_{\mathrm{D}}$ causes an increase of the total decay rate $\Gamma_{\mathrm{T}}$ measured in a time resolved experiment. Thus, measuring the lifetime of the donor in the absence $\left(\Gamma_{\mathrm{D}}\right)$ and presence $\left(\Gamma_{\mathrm{T}}=\Gamma_{\mathrm{D}}+\Gamma_{\mathrm{FRET}}\right)$ of acceptor yields the FRET decay rate. Once this is known, the efficiency of FRET is given by the ratio between the decay rate associated with FRET and the total rate. 

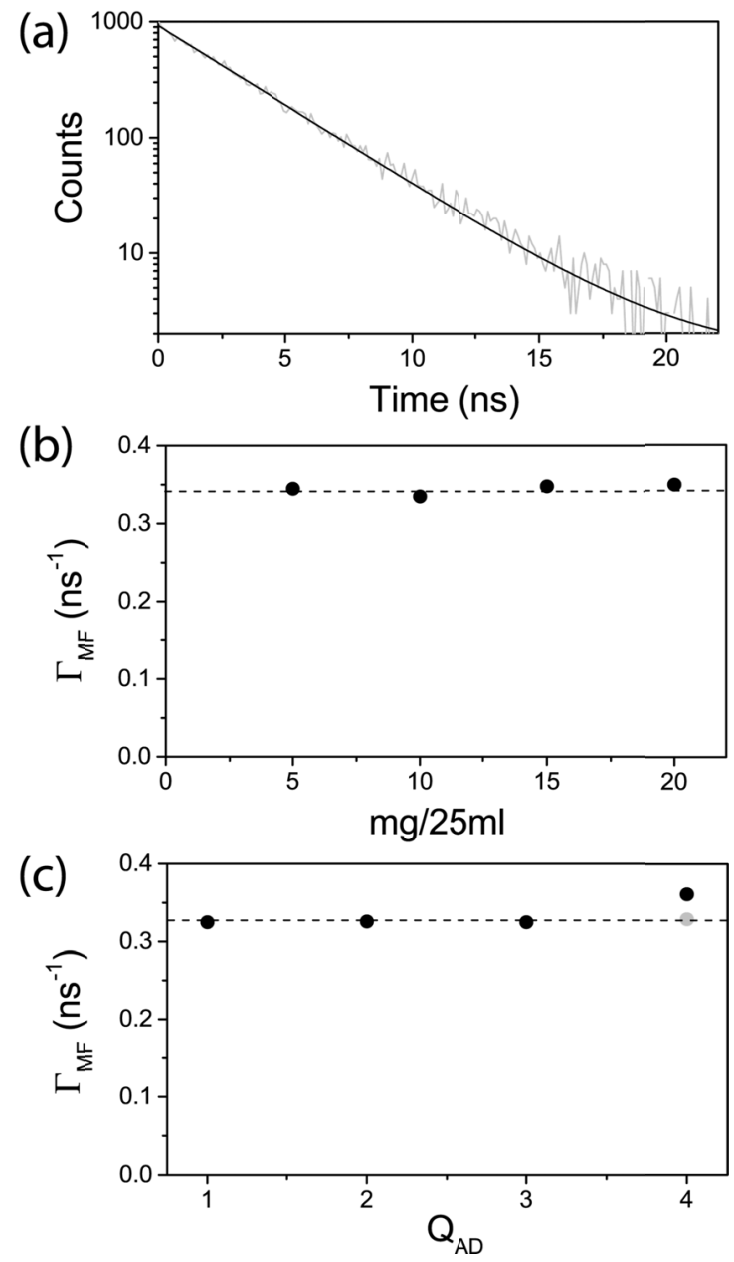

Fig. 5 (a) PL decay curve for a dispersion of PMMA spheres containing only RhB (grey) and fit with a lognormal distribution of decay rates (black curve). (b) Most frequent value $\Gamma_{M F}$ of the rate distribution as a function of dye concentration for PMMA spheres fabricated using $25 \mathrm{ml}$ of MMA containing RhB only. (c) $\Gamma_{M F}$ associated with the decay of Rh700 measured for spheres containing RhB and Rh700 (grey dot corresponds to a reference sample having the same acceptor concentration as $Q_{A D}=4$ only with no donor).

PL decay curves at the donor's emission $(\lambda=585 \mathrm{~nm})$ were measured for samples having different $Q_{\mathrm{AD}}$ ratios when pumped with $\lambda=515 \mathrm{~nm}$. Figure 6 shows results for spheres having a diameter of $380 \mathrm{~nm}$. As acceptor molecules are included within the spheres the decay curves become steeper pointing to the introduction of non-radiative (FRET) de-excitation paths. Furthermore, similar to other polymeric matrices such as DNA-CTMA complexes, [28] the curves become strongly multiexponential which indicates that an increasing number of donor/acceptor configurations exist. In the present case the distribution of FRET processes has a twofold origin: on the one hand a distribution of D/A separations and orientations as they are incorporated into the polymeric matrix. On the other hand a radial concentration gradient exists due to the fact that the amount of dye in the reaction increases as the polymerization takes place (see Experimental section). 


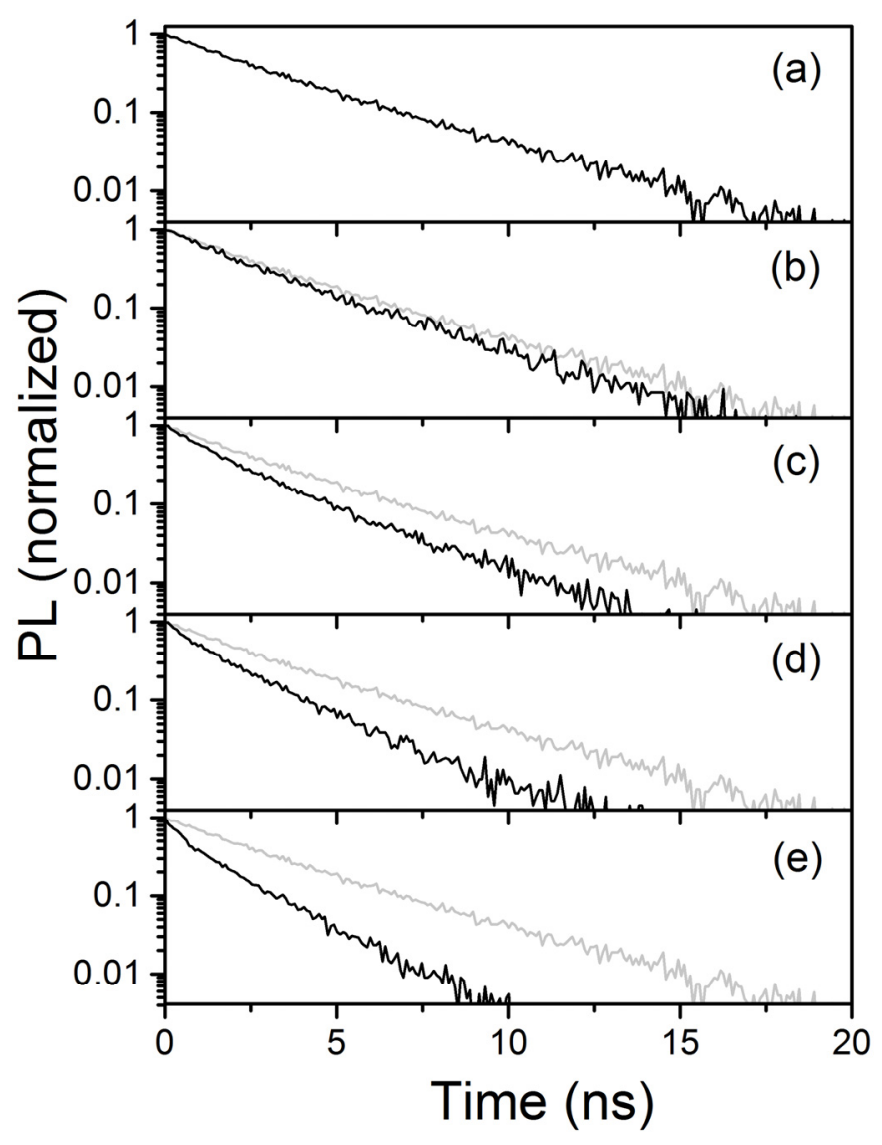

Fig. 6 PL decay curves measured for spheres with a diameter of $380 \mathrm{~nm}$. (a)-(e) correspond to $Q_{A D}=0-4$. Grey curve shows the result for $Q_{A D}=0$ and is shown for comparison.

In order to gain further insight into the FRET process within the PMMA spheres as the $Q_{\mathrm{AD}}$ ratio increases we will consider the decay distribution extracted from the fitting of the experimental curves. Figure 7 shows lognormal distributions $\rho(\Gamma)$ for the different values of $Q_{\mathrm{AD}}$ considered in the present work as derived from the PL decay curves. Here we can see how as the acceptor concentration increases for a fixed amount of donor molecules the distribution shifts to larger $\Gamma$ values, corresponding to an overall faster decay. Since no aggregation between the molecules is taking place as mentioned above, this corresponds to the introduction of additional non-radiative decay (FRET) paths. Further, as the distribution shifts to larger values it becomes broader, evidencing the existence of an increasing number of D/A configurations. The latter likely comprises both separations and orientations as no preferred molecule orientation is expected to take place within the polymeric matrix. 


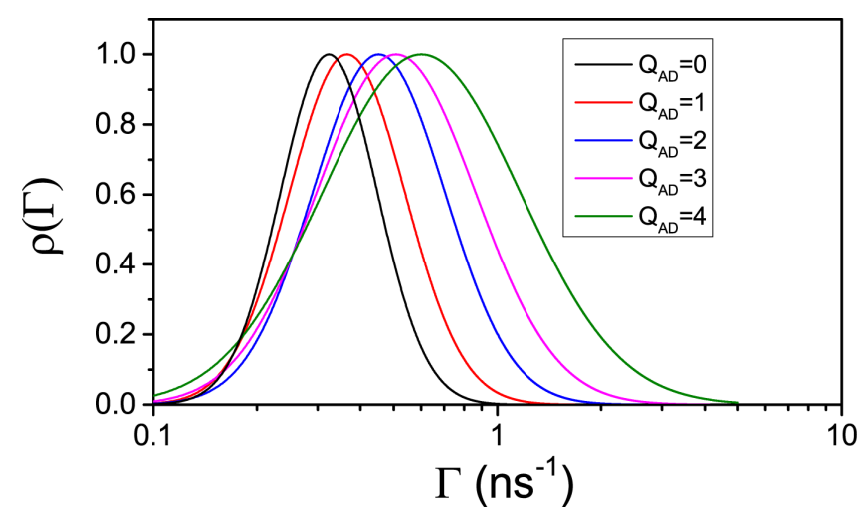

Fig. 7 Distribution of decay rates extracted from fitting PL decay curves with a lognormal function.

From the most frequent value $\left(\Gamma_{\mathrm{MF}}\right)$ of the lognormal distribution which best fits the data we can extract an effective FRET efficiency $\eta_{\mathrm{eff}}=\Gamma_{\mathrm{FRET}} /\left(\Gamma_{\mathrm{FRET}}+\Gamma_{\mathrm{D}}\right)=\left(\Gamma_{\mathrm{MF}}-\Gamma_{\mathrm{D}}\right) /\left(\Gamma_{\mathrm{MF}}\right)$. [28] Figure 8 shows the effective efficiency for samples with three different sphere diametters: 250,380 and $480 \mathrm{~nm}$. From the graph it is evident that the smaller the spheres, the larger the efficiency achieved for a given $Q_{\mathrm{AD}}$ ratio. This could indicate that the average separation between donor and acceptor molecules within the sphere decreases with its diameter, in agreement with the fact that the ratio between the amount of dye added to a reaction and the MMA volume for a given $Q_{\mathrm{AD}}$ increases as we decrease the sphere diameter. For the three samples shown, maximum efficiencies of $c a .40 \%$ were observed before concentration quenching took place (see above) for $Q_{\mathrm{AD}}$ ratios of 1 and 3 for the 250 and $480 \mathrm{~nm}$ spheres respectively. In parallel, the fit to a lognormal distribution yielded good results (with $\chi_{\mathrm{N}}{ }^{2}$ in the 1-2 range) up to the $Q_{\mathrm{AD}}$ value for which quenching takes place, for which the fit worsens. This indicates that the way the dyes are arranged within the polymeric matrix and their nature (their spectral properties) preclude a larger FRET before concentration quenching takes place. This in principle could be improved with a different choice of dyes presenting a larger spectral overlap, where the same FRET efficiency could be achieved with a larger separation between donors and acceptors.

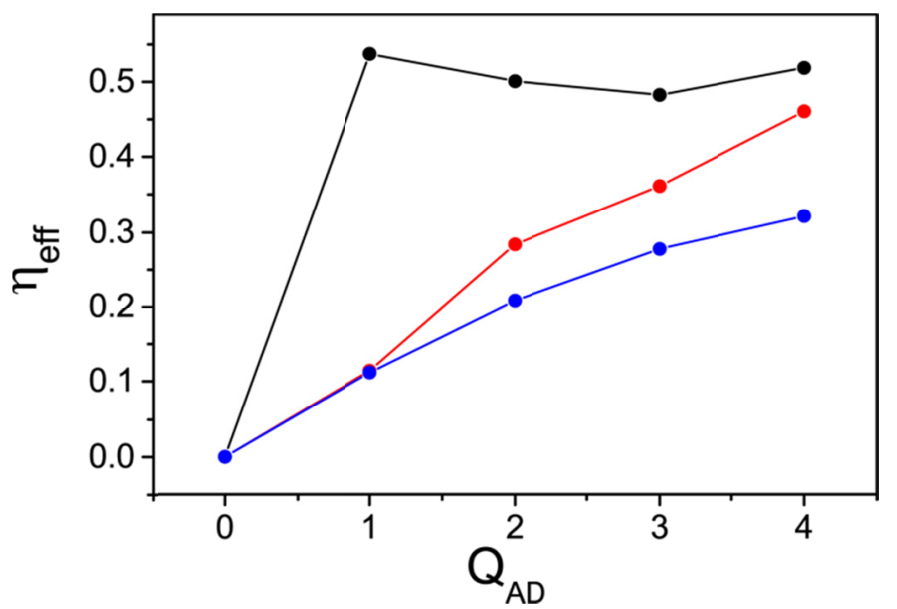

Fig. 8 Effective FRET efficiency as a function of $Q_{A D}$ extracted from the decay dynamics of samples having different sphere size: 250 (black), 380 (red) and 480 nm (blue). 
To further evidence the quality of the spheres, $3 \mathrm{D} \mathrm{PhC}$ in the shape of thin film artificial opals (see Fig. 9) were fabricated following the vertical deposition method [30] using previously reported [31] experimental conditions. Figure 9c shows normal incidence reflectance spectra measured on $350 \times 350 \mu \mathrm{m}$ regions of samples made from spheres of different diameter. The large reflectance peak, associated with Bragg diffraction by the (111) planes parallel to the sample surface, redshifts with increasing sphere size corresponding to a larger lattice parameter. Two evidences of the high quality of the fabricated structures are the high ( $\geq 50 \%)$ reflectivity achieved and the presence of secondary oscillations surrounding the main Bragg peak. The latter correspond to Fabry-Perot oscillations associated with interference between light reflected at the air-sample and sample-substrate interfaces for wavelengths out of the Bragg diffraction condition. [32] The similar reflectance for samples fabricated with different sphere sizes is in agreement with the small and nearly constant sphere polydispersity reported (see Fig. 2).

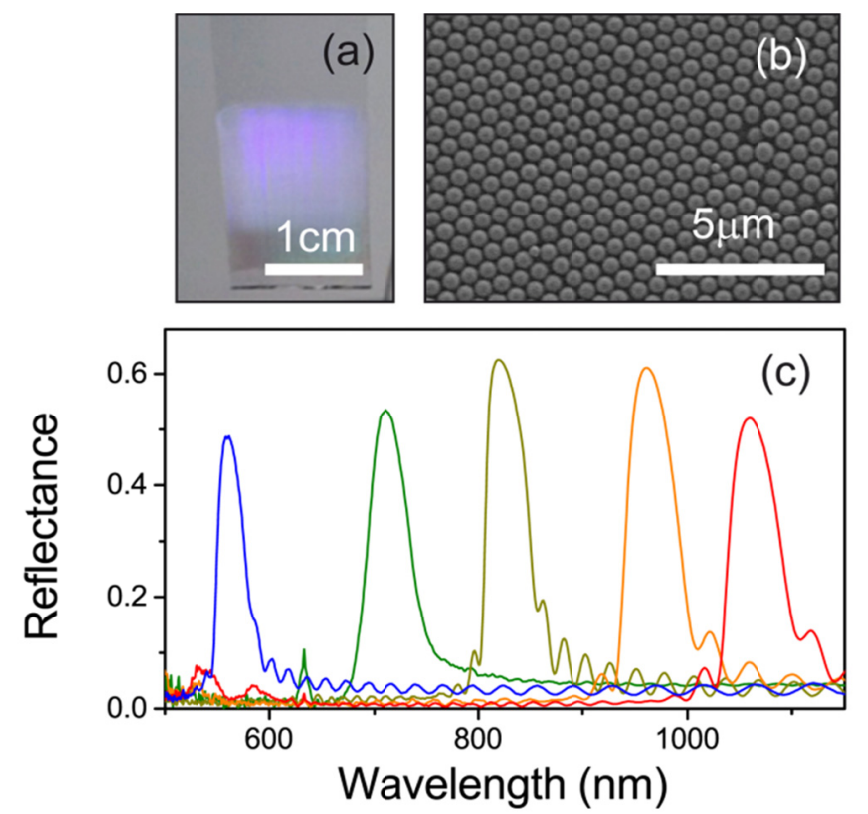

Fig. 9 (a) Photograph of an artificial opal fabricated on a glass substrate from spheres having a diameter of $475 \mathrm{~nm}$. (b) SEM image of the surface of the same sample. (c) Normal incidence reflectance for samples fabricated from spheres having different diameters. Left to right: 250, 320, 380. 430 and $475 \mathrm{~nm}$.

Finally, we explored the possibility of modifying the energy transfer between the FRET pair within the spheres once incorporated into the photonic structures. In particular we considered the sample having the smallest diameter $(250 \mathrm{~nm})$ for which the Bragg peak at normal incidence matches the donor emission (see Fig. 10). For the case of acceptor to donor ratio $Q_{\mathrm{AD}}=2$ the PL of the sample was collected at normal incidence with a microscope objective $(\mathrm{NA}=0.75)$ which also served to focus the pump beam onto the sample. With a tunable iris aperture placed after the objective and before the spectrometer we could select the collection angular range. For collection close to normal incidence (angular range $\alpha=6^{\circ}$ ) the PL from the acceptor is much larger than the donor PL as compared to the emission of the spheres in solution (see Fig. 10b). In order to understand the origin of these changes PL decay measurements were carried out. No significant difference in the donor $\Gamma$ was observed for spheres in solution and in the PhC, excluding a profound modification of the FRET efficiency. Rather, the observed changes are attributed to a filter effect where Bragg diffraction by the (111) planes precludes the donor emission from exiting the sample at normal incidence. This was further confirmed by increasing the collection angular range to $48^{\circ}$ (the maximum allowed by the objective's NA) and 
measuring PL becoming even closer to the reference one corresponding to spheres in solution.. The absence of changes in the FRET efficiency is likely due to the low refractive index contrast in these samples. Nevertheless, these structures are amenable to be further infiltrated with large refractive index oxides (such as $\mathrm{TiO}_{2}$ ) with transparency in the emission range of the dyes and thus larger changes in LDOS.

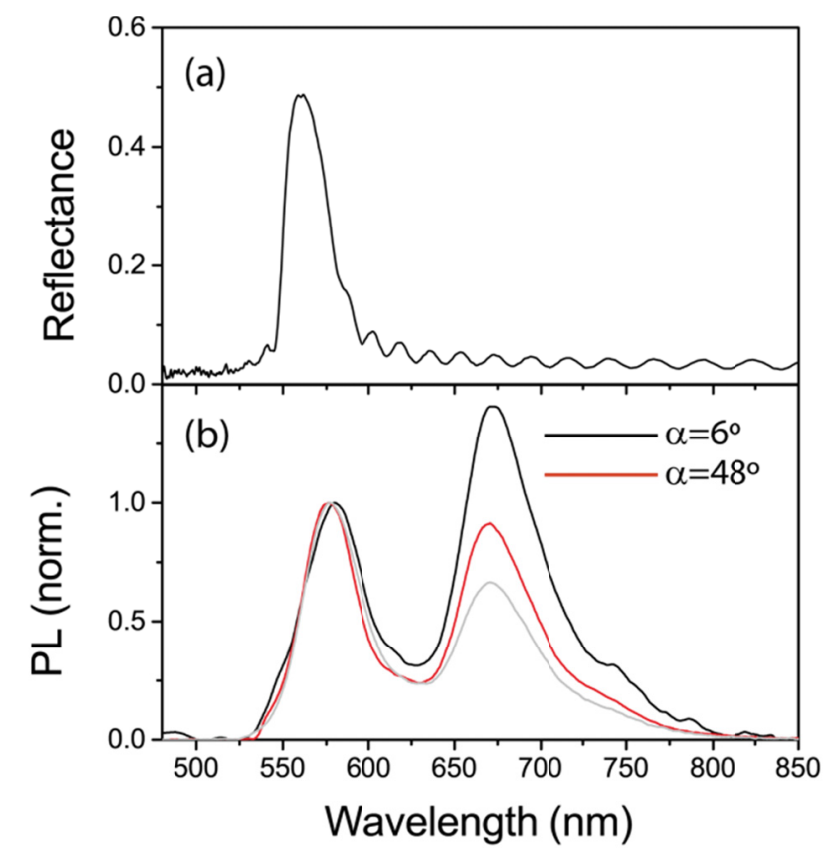

Fig. 10 (a) Reflectance spectrum for an artificial opal made from 250nm spheres having a $Q_{A D}=2$. (b) PL spectra collected at normal incidence with a collection angle of $6^{\circ}$ (black) and $48^{\circ}$ (red) as well as PL from spheres in solution (grey curve).

\section{Conclusions}

We have presented a method to fabricate highly monodisperse submicron polymeric spheres containing a FRET pair consisting of two rhodamine dyes (RhB and Rh700). An optical characterization by means of PL spectroscopy and time resolved measurements has allowed us to extract the efficiency of the energy transfer taking place between the donor and acceptor. By changing the donor/acceptor ratio the FRET efficiency could be modified up to a limit of $c a$. $40 \%$ where concentration quenching effects appear. The low polydyspersity of the spheres $(\mathrm{ca}$. $3 \%$ ) allowed for the fabrication of high quality 3D self-assembled $\mathrm{PhC}$ in the form of artificial opals. Further, with the proper sphere diameter the Bragg peak of the $\mathrm{PhC}$ could be matched with the donor's emission allowing for a modification of the PL of the spheres through a filter effect caused bt Bragg diffraction by the planes of spheres parallel to the sample surface, which introduces an additional path to tune the sphere's emission. The present luminescent beads could be used in the future as a platform to fabricate photonic structures with a higher refractive index contrast where the efficiency of the FRET process could be modified through the local density of radiative states.

\section{Acknowledgement}

This work was supported by the Spanish MINECO MAT2012-31659 (SAMAP) projects; Comunidad de Madrid S2013/MIT-2740 (PHAMA_2.0) programme and EU Nanophotonics4Energy contract 248855. 


\section{References}

[1]T. Förster, Ann. Phys. 1948, $2,55$.

[2 L. Wang, T. Weihong, Nano Lett. 2006, 6, 84.

[3]J. Fölling, S. Polyakova, V. Belov, A. van Blaaderen, M.L. Bossi, S.W. Hell, Small 2008, 4, 134.

[4]L. Zhu, W. Wu, M-Q. Zhu, J.J. Han, J.K. Hurst, A.D.Q. Li, J. Am. Chem. Soc. 2007, 129, 3524.

[5]W-B. Wu, M-L. Wang, Y-M. Sun, W. Huang, Y-P. Cui, C-X. Xu, Opt. Mater. 2008, 30, 1803.

[6]C. Wu, Y. Zheng, C. Szymanski, J. McNeill, J. Phys. Chem. C 2008, 112, 1772.

[7]J. Chen, P. Zhang, G. Fang, P. Yi, F. Zeng, S. Wu, J. Phys. Chem. B 2012, 116, 4354.

[8]W.J. Rieter, J.S. Kim, K.M.L. Taylor, H. An, W. Lin, T. Tarrant, W. Lin, Angew. Chem. Int. Ed. 2007, 46, 3680.

[9]E. Rampazzo, S. Bonacchi, R. Juris, M. Montalti, D. Genovese, N. Zaccheroni, L. Prodi, D.C. Rambaldi, A. Zattoni, P. Reschiglian, J. Phys. Chem. B 2010, 114, 14605.

[10] H. Choi, N. Cho, S. Paek, J. Ko, J. Phys. Chem. C 2014, 118, 16319.

[11] J.S. Huang, T. Goh, X. Li, M.Y. Sfeir, E.A. Bielinski, S. Tomasulo, M.L. Lee, N. Hazari, A.D. Taylor, Nat. Photonics 2013, 7, 479.

[12] B. Bao, F. Li, H. Li, L. Chen, C. Ye, J. Zhou, J. Wang, Y. Song, L. Jiang, J. Mater. Chem. C 2013, 1, 3802.

[13] M. A. Baldo, M. E. Thompson, S. R. Forrest, Nature 2000, 403, 750.

[14] L. Cerdán, E. Enciso, V. Martín, J. Bañuelos, I. López-Arbeloa, A. Costela, I. GarcíaMoreno, Nat. Photonics 2012, 6, 621.

[15] C. Blum, N. Zijlstra, A. Lagendijk, M. Wubs, A. Mosk, V. Subramaniam, W.L. Vos, Phys. Rev. Lett. 2012, 109, 203601.

[16] J.F. Galisteo-López, M. Ibisate, R. Sapienza, L.S. Froufe-Pérez, A. Blanco, C. López, Adv. Mater. 2011, 23, 30-69.

[17] L. González-Urbina, K. Baert, B. Kolaric, J. Pérez-Moreno, K. Clays, , Chem. Rev. 2012, 112, 2268.

[18] B. Kolaric, K. Baert, M. Van der Auweraer, R.A.L. Vallée, K. Clays, Chem. Mater. 2007, 19, 5547.

[19] Z. Yang, X. Zhou, X. Huang, J. Zhou, G. Yang, Q. Xie, L. Sun, B. Li, Opt. Lett. 2008, 33, 1963.

[20] Z. Yang, X. Huang, L. Sun, J. Zhou, G. Yang, B. Li, C. Yu, J. Appl. Phys. 2009, 105, 083523.

[21] M. Li, F. He, Q. Liao, J. Liu, L. Xu, L. Jiang, Y. Song, S. Wang, D. Zhu, Angew. Chem. Int. Ed. 2008, 47, 7258.

[22] H. Li, J. Wang, H. Lin, L. Xu, W. Xu, R. Wang, Y. Song, D. Zhu, Adv. Mater. 2009, 22, 1237.

[23] A.F. Koendrink, A. Lagendijk, W.L. Vos, Phys. Rev. B 2005, 72, 153102.

[24] S-E. Phan, W.B. Russel, J. Zhu, P.M. Chaikin, J. Chem. Phys. 1998, 108, 9789.

[25] S. Furumi, H. Fudouzi, T. Sawada, Las. Phot. Rev. 2009, 4, 205. 
[26] M. Berggren, A. Dodabalapur, R.E. Slusher, Z. Bao, Nature 1997, 389, 466.

[27] Lakowicz J. R. (Ed.) Topics in Fluorescence Spectroscopy Vol. 2; Kluwer Academic Publishers: New York, 1973.

[28] M. Ibisate, J.F. Galisteo-López, V. Esteso, C. López, Adv. Opt. Mater. 2013, 1, 651.

[29] R. Sapienza, P. Bondareff, R. Pierrat, B. Habert, R. Carminati, N.F. van Hulst, Phys. Rev. Lett. 2011, 106, 163902.

[30] P. Jiang, J. F. Bertone, K. S. Hwang, V. L. Colvin, Chem. Mater. 1999, 11, 2132.

[31] J. F. Galisteo-López, E. Palacios-Lidón, E. Castillo-Martínez, C. López, Phys. Rev. B 2003, 68, 115109.

[32] Yu. A Vlasov, M. Deutsch, D. J. Norris, Appl. Phys. Lett. 2000, 76, 1627. 